\title{
Socrate, lon et la Poésie. La structure dialectique de l'Ion de Platon
} Marie Delcourt

\section{Citer ce document / Cite this document :}

Delcourt Marie. Socrate, Ion et la Poésie. La structure dialectique de l'Ion de Platon. In: Bulletin de l'Association Guillaume Budé, ${ }^{\circ} 55$, avril 1937. pp. 4-14;

doi : 10.3406/bude.1937.6118

http://www.persee.fr/doc/bude_0004-5527_1937_num_55_1_6118

Document généré le 17/03/2016 


\section{SOGRATE, ION ET LA POÉSIE}

\section{LA STRUGTURE DIALEGTIQUE DE L'ION DE PLATON}

Ion ${ }^{1}$ donne sur la poésie et sur l'art du rhapsode des détails qui sont incohérents seulement dans la forme. Socrate n'a aucune peine à les ramener à une doctrine ferme qui est aussi la sienne, à savoir que, dans la création poétique el dans l'exégèse poétique, la raison n'est pour ricn. L'irrationalité de la poésie nous apparaît comme une thèse romantique. De même que Socrate, les romantiques font de l'inspiration quelque chose d'inexplicable, une sorte de cadeau d'un dicu ${ }^{2}$. Seulement, pour Socrate, la poésie est infra-rationnelle; pour les romantiques - et pour Ion - elle est suprarationnelle. Cette différence d'appréciation est simplement impliquée dans le dialogue. Elle résulte pour nous de ce que nous savons par ailleurs de la philosophie de Socrate, fondée sur la primauté de la raison et sur le privilège des connaissances communes à tous les hommes, exprimées à l'aide d'une

I. Ion est un homme qui récile des vers et qui les explique : le

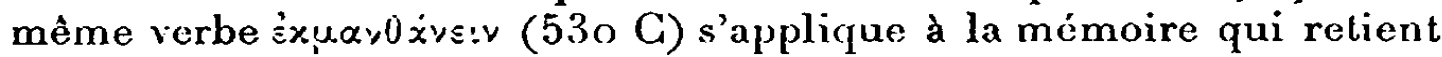
les textes (

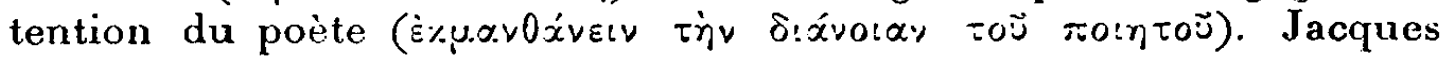
Gopeau, lecteur, acteur et exégète, représenterait assez exactement une incarnation moderue du rhapsode hellénique.

2. La thèse classique est vigourensement plaidée, à propos de l'acteur, proche parent du rhapsode, par Diderot dans le Paradoxe sur le Comédien. A propos du poète, Edgar Poö passe à la limite dans The philosophy of composition. 
langue unique, avec un minimum de différences individuelles. Les mathématiques, qui rapprochent tous les esprits dans un parfait accord intellectuel ${ }^{1}$, sont intrinsèquement supérieures à la poésie qui isole, puisqu'elle exprime un individu ct n'exprime que lui. Le fait que l'intelligence poétique est une correspondance de singulier à singulier est symbolisé par Platon sous une forme un peu caricaturale par le choix même du personnage d'Ion, qui s'est cantonné une fois pour toutes dans la lecture d'Homère et qui suit avec peine une discussion logiquement ordonnée. L'impuissance dialectique d'Ion est destinée à suggérer que l'homme qui se voue à la connaissance poétique est inférieur à celui qui procède selon les règles de la raison, car le second comprend le premier et la réciproque n'est pas vraie.

Toutefois, les commentateurs ont tellement exagéré ce qu'ils appellent la " bètise », la « stupidité » d'Ion qu'il est bon d'y regarder de plus près ${ }^{2}$. Ion n'est nullement un imbécile. C'est simplement un homme qui n'a pas l'habitude de la joute dialectique et qui fait des concessions imprudentes. Socrate les exploite aussitòt en parfait sophiste. Voyons quelles sont ces concessions et comment Socrate en profite.

$$
\text { ** } *
$$

Ion commence par affirmer qu'il comprend Homère mieux que personne, mais qu'il n'entend rien ni à Hésiode, ni à Archiloque. Cette position n'est absurde que par son outrance. 'Tout critique peut dire (et dit en effet sans que personne

r. Ménon 82-86 et surtout $85 \mathrm{E}$. Du mème fond la distinction des trois langages dans l'Eupalinos de Valéry, p. i $45 \mathrm{sqq}$. de la i $\mathrm{I}^{\mathrm{e}}$ éd.

2. On ne saurait assez déplorer le ton sentimental adopté par la plupart des commentateurs lorsqu'il est question de Socrate. A les en croire, il aurait toujours raison. Ils plaident aussi chaleureusement que s'il ćtait en leur pouvoir d'épargner la ciguë au condamné de 399. Malheureusement leurs étudiants ne les croient pas et se dégoùtent d'une lecture au cours de laquelle on ne cesse de forcer leur adhésion. 
songe à s'en étonner) qu'il renonce à parler de telle ou telle ouvre parce qu'il sent manquer, entre elle et lui, les correspondances nécessaires. Et ces correspondances, à son regard et au regard d'autrui, proviennent généralement de données impondérables, d'inexplicables sympathies ${ }^{1}$. Seulement, un critique de métier, un comédien de métior n'excluent un poète qu'à titre d'exception. Ion, au contraire, exclut tous les poètes : son intuition joue au bénéfice d'Homère seul et il s'en fait gloire. La transposition caricaturale résulte d'un « passage à la limite », procédé familier à Platon.

Socrate pose alors une question-piege: « $Y$ a-t-il un sujet sur lequel Homère et Hésiode parlent de même ${ }^{2}$ ? 》 Ion, avec une surprenante rapidité, répond affirmativement. Réponse étonnante pour un lecteur moderne qui serait bien empêché de ciler un seul sujet commun aux deux poètes, un seul passage où ils se rencontrent. Réponse maladroite, car elle va autoriser Socrate à identifier le poème avec la somme de ses matériaux et, par conséquent, à préparer, entre le poìme un et achevé d'une part, et ses éléments dissociables d'autre part, une confusion où Ion finira par s'embourber. Socrate pousse aussitót son avantagre et demande : $I^{\circ}$ si dans un de ces «passages communs », Ion comprend Hésiode aussi bien qu'Homère. Ion, qui répond oui, devrait répondre non et refuser de laisser confondre le poìme avec la somme de ses matériaux; $2^{\circ}$ si, dans le cas où les poìtes sont en désaccord

I. Arsène Sorcil, Introduction à l'histoire de l'esthétique francaise, Bruxelles, I930, a parfaitenent décrit l" "hédonisme honteux 》 des classiques. An fond, ils pensent commo Montaigne : "Si quelqu'un me dit que c'est avilir les lluses de m'en servir seulement de jouct et de passe-temps, il ne sait pas, comme moi, combien vaut le plaisir, lo jou et le passe-tomps : a peine quo je ne dise toute autre fin citre ridicule " (cité par Soreil, op. cil., p. 18); mais lous ne l'avouent pas ; ils s'obstinent' à tenir l'art du côté de la raison et même de la philosophie et ils admirent Homère d'ètre un savant universel.

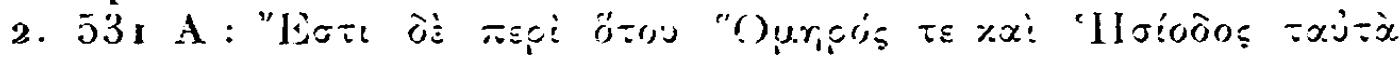
$\lambda \hat{s}_{y} \varepsilon \tau^{\circ}$; Je ne vois pas qu'aucun critique se soit interrogé sur la nature de ces coïncidences entre Homire at Hésiode. 
au sujet, par exemple, de la divination, lon est aussi compétent qu'un devin pour les départager. Ion reconnaît son infériorité. La confusion entre le poème et la somme de ses éléments, imprudemment acceptée par lui, est dès maintenant acquise.

Mais, dit Socrate, tous les poètes mettent en cuvre le même matéricl, à savoir les relations, affections et sentiments des hommes et des dieux ${ }^{1}$. Or lorsqu'il s'agit d'une science, c'est-à-dire d'un département de la connaissance qui étudie un matériel in telligible homogène, un seul et même "savant » est juge de la vérité et de l'erreur, de l'excellence et de la médiocrité. Comment Ion peut-il donc soutenir à la fois qu'Homère parle mieux qu'Hésiode et que lui, Ion, entend Ilomère mais non Ilésiode? En effet, Ion a cornmis là une nouvelle imprudence: pour rester dans son système, qui consiste à reconnaître entre le poète et le critique uniquement une correspondance subjective et toute irrationnelle, il n'aurait pas dû dire qu'Homère parle mieux qu'llésiode, car cela revient à dresser une série dont le « savant » est capable de juger chaque tcrme, puisque ce jugement est logiquement antérieur à l'établissement de la série. Il aurait dù dire qu'Ilomère parle autrement qu'Hésiode et que la singularité d'Homère correspond à sa singularité personnelle à lui, Ion. Cette distinction, qu'il ne formule pas, est clairement impliquée dans sa réponse à Socrate $\left(53_{2} \mathrm{~B}\right)$ : Tout se que je sais, c'est que les autres poètes m'emdorment et qu'llomère m'intéresse. Socrate consent provisoirement à s'en tenir à ce subjectivisme et conclut: Dans ce cas, ce que tu fais ne relève

I. Il est impossible de prendre oúpávı $\tau_{\alpha} \alpha \gamma_{i}^{\prime} \mu \alpha \tau \alpha$ (53 I Ci) dans lo sens de " pbénomènes célestes" comme le veulent cortains commentateurs qui rappcllent que Métrodore de Iampsaque lisait dans Homère une physique du monde. C'est introduire une disparate dans un passage très homogène. L'expression se trouve dans un contexto où il est uniquement quostion d'entités morales; clle est opposóc à $\tau \dot{x} \dot{s}\rangle$ "A

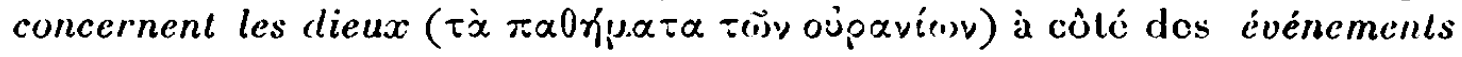
qui concernent les morts. 
pas d'une science, car une science connaît à la fois toute une série homogène. Ett tu reconnais, n'est-ce pas, qu'il y a un ensemble qui est la poésie comme il y a un ensemble qui est l'art du dessin 1 ?

Ici encore, Ion répond oui alors qu'en bonne logique, il devrait répondre non. Dansson systèmeil n'y a pas de « science poétique »; il n'y a que des poètes; de même qu'il n'y a pas de science rhapsodique; il n'y a que des rhapsodes. Les correspondances s'établissent en vertu d'affinités individuelles dont lui-même a signalé le caractère subjectif. Ce caractère, il devrait le souligner maintenant. De même, lorsque Socrate lui demande $(532 \mathrm{E})$ s'il y a des gens qui comprennent tel peintre, tel musicien, à l'exclusion des autres ${ }^{2}$, il devrait répondre affirmativement et reconnaitre l'irrationalité de notre compréhension en présence des arts du dessin, des arts des sons, comme l'irrationalité de notre compréhension en prósence des arts de la parole. Mais il ne va pas jusqu'à de telles généralités : personne plus qu'Ion n'a de répugnance à dépasser l'individuel. Il se borne à répéter que lui comprend parfaitement Homère et Homère seul.

C'est Socrate qui tire la conclusion : l'intelligence de la poésie ne peut s'expliquer si l'on ne remonte d'abord à la nature de la poésie elle-mème. Celle-ci résulte d'une inspiration divine qui va de la Muse au poète; la connaissance de la poésie en est une émanation qui va du poète à l'interprète.

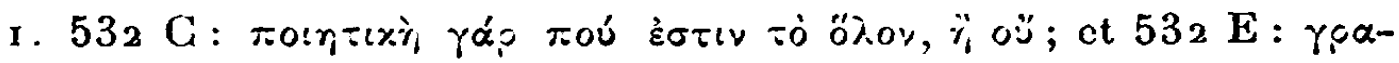
Q̣:

2. Pourquoi Platon mentionne-t-il, après Polygnote, une série de sculpteurs très anciens: le mythique Dédale, l'homérique Epéos qu'A théna aida à faire le cheval de Troie, et Thćodore de Samos, soit le ciseleur des offrandes de Crésus, soit le joaillier de Polycrate? Ses lecteurs avait pu voir le célèbre tableau de Polygnote à Delphes, mais aucuu travail d'Epéos ni de Dédale. De mẻme, pourquoi ne citer, parmi les musiciens et rhapsodes, que deux personnages mythiques, Olympos et Orphée, et deux personnages homériques, Thamyros et Phémios? Et, plus bas, à côté d'Ilomère, Orphée et Musće ? Il y a là une intention dont la portée nous échappe. 
Une nouvelle émanation va de l'interprète au public. Ce qu'Ion n'a pas su ou n'a pas osé dire, c'est Socrate qui le dit, à savoir qu'il n'y a pas un tout qui s'appelle la poésie, mais qu'il y a seulement des relations individuelles; il n'y a pas, parmi les poc̀tes et parmi leurs interprètes, des séries linéaires sur le long desquelles sont situées l'erreur et la vérité, mais un rayonnement qui s'affaiblit et se dégrade entre le centre et la périphérie. Il n'y a pas du meilleur et du moins bon, mais du différent. Meilleur, dirions-nous en jargon moderne, est simplement un camouflage rationaliste pour préféré. Le poète agit, non pas conformément aux lois de l'intelligence, mais conformément aux lois d'un instinct qui lui est propre. Il va comme l'abeille, poussé par la nature, sans savoir où il va ${ }^{1}$. Entre le poète et le poème, la loi de causa-

I. La comparaison avec l'abeille est bientôt devenue célèbre, mais elle a entraîné un glissement de sens. Platon voit ici dans l'abeille l'animal léger guidé par un instinct irrationnel. Mais elle est aussi la butineuse patiente qui compose le miel. Horace, s'opposant à Pindare, cygne de Dircé, se donne comme une abeille laborieuse qui fait les vers à force de travail (Odes, IV, 2, 25 sqq.). Il pense certainement à l'image évoquée par Platon, mais pour en tirer une idée qui est en contradiction complète avec celle que Platon a voulu suggérer. La Fontaine, comme Horace, se sert de l'image de l'abeille pour désigner le poète qui imite et qui imite plusieurs maîtres, - c'est-àdire qui travaille en vertu d'une esthétique qui est anx antipodes de celle de Platon :

Sur différentes fleurs l'abeille se repose,

Et fait du miel de toutes choses

(Disc. à $M^{\text {me }}$ de la Sablière, Fables $\mathrm{X}, \mathrm{I}$.)

Lorsqu'il veut insister sur son insoumission à la raison, il ajoute à l'image de l'abeille celle du papillon :

Papillon du Parnasse et semblable aux abeilles

A qui le bon Platon compare nos merveilles...

(Disc. a $M^{\text {me }}$ de la Sablière, dans les Poésies diverses.)

Au surplus, si l'on veut mesurer l'écart qu'il y a entre l'esthétique de Platon et celle de la Fontaine, qu'on se souvienne que Socrate pose en principe que la Muse attire un poète vers un scul genre 
lité ne joue pas (exemple de Tynnichos): un homme médiocre peut écrire un bel ouvrage, à condition qu'il sorte de luimême ct se laisse habiter par le flux divin.

Sur tout ceci, Socrate-Platon et Ion sont entièrement d'accord. Mais chacun d'eux met l'accent sur un élément différent de la délinition. Socrate voit ce dont l'homme s'appauvrit en se vidant de sa raison : Ion voit cc dont il s'enrichit en se laissant traverser par le fluide qui rayonne de la Muse ${ }^{1}$. Aussi proteste-t-il lorsque Socrate se met à insister sur le cỏté négatif de la définition: Tu devrais être bien habile pour arriver à me persuader que je suis en état de transe et de folie quand je loue Homère $(536 \mathrm{D})$. Il accepte la théorie du «lot divin »dans ce qu'elle a de flatteur, non dans ses autres conséquences.

Socrate revient alors, pour en tirer parti, à la plus impru(53/4) (andis que La Fontaine dit:

J'irais plus haut peut-être au temple de Nímoirc

Si dans un genre seul j'avais usé mes jours,

Mais quoi $?$ je suis volage en vers comme en amour.

C'est sa qualité d'abeille butineuse qui l'a amené à se disperser comme il l'a fait. La comparaison moderne qui se rapproche le plus do l' " abeille " d'Ion, c'est Ie "Ich singe, wie der Vogel singt » de Gothe, qui traduit la mème idée, avec une autre image.

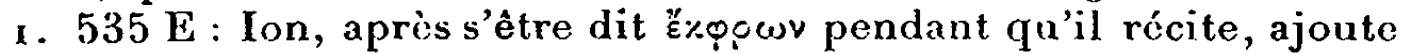
qu'il suil des yeux les effets de ses paroles sur le visage de ses auditeurs : "Il faut bien que j'y prenne garde, car, si je les amène ̀̀ pleurer, c'est moi qui rirai en recevant mon argent ; s'ils rient de moi, c'est moi qui pleurerai quand on me refusera mon salaire. "

D'anecdotes de ce genre, Diderot tire argument pour montrer que

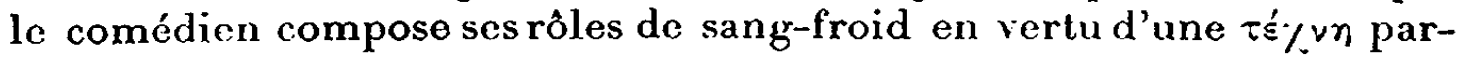
faitcment rationnelle. Il y a certes, entre les deux déclarations d'Ion, uno contradiction que Socrate no relève point. C'est, ou bien que ce n'est pas là qu'il désire prendre Ion en faute; ou bien qu'il ne veut pas toucher à la thèse de l'irrationalité en cascade du don poćtique et de ses succédanés; ou bien qu'il estime que la chose est explicable, étant donné que la raison du rhapsode, congédiće du travail rhapsodique seul, peut travailler néanmoins sur des questions vul_ gaires ; ou encore que Platon, dans son désir d'accentuer la caricature, a introduit un trait ridicule sans s'apercevoir qu'il fait disparate. 
dente des concessions d'Ion: l'identification du poème avec la somme de ses éléments, et il rappelle un principe dont lui et Ion étaient convenus à ce moment : tous les objets de même nature relèvent d'une science unique. Il ajoute maintenant : et une science unique ne connaît qu'une série homogène ; puis ceci qui est évidemment faux : qu'un objet quelconque $^{1}$ ne peut relever que d'une science unique $(537 \mathrm{D}$ :

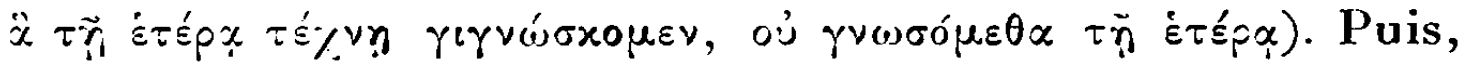
relevant dans Homère un passage relatif au pilotage du char, un autre à la médecine, un autre à la pêche, un autre à la divination, il amène Ion à avouer qu'il appartient au cocher, au médecin, au pècheur, au devin d'apprécier l'exactitude de ces descriptions. Mais alors, quelle est la compétence du rhapsode qui se trouve évincé de chaque paragraphe du texte et remplacé par un autre technicien? Ion, qui se rend parfaitement compte que la thèse de Socrate est insoutenable, mais qui est incapable de la réfuter correctement, répond avec l'énergie du désespoir que le rhapsode reste juge de tous les éléments de l'ouvre :

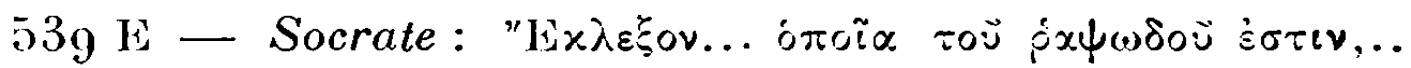

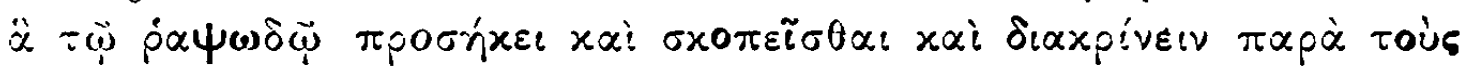

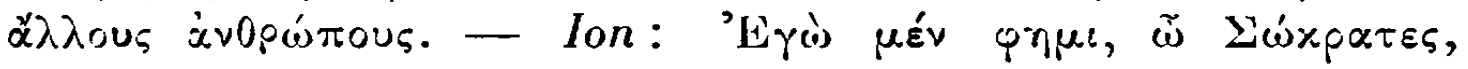
$\ddot{\alpha} \pi \alpha \nu \tau \alpha$.

Il est évident qu'Ion a raison, mais il s'exprime mal. Au lieu de répondre $\alpha^{\prime} \pi x v \tau \alpha$ - et Socrate, bien entendu, va le prendre tout de suite en flagrant délit de contradiction, puisqu'ils sont convenus d'accorder tels vers au médecin, tels vers au divin et ainsi de suite, -- il devrait dire : $\tau \grave{o} \pi \ddot{\alpha} v, x \alpha \tau \dot{\alpha}$

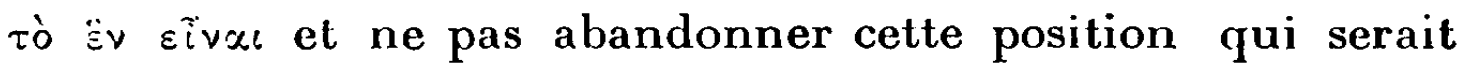
inexpugnable, puisqu'elle établirait l'existence d'un objet nouveau, le poème, supérieur ou au moins hétérogène à la

$x$. Le contexte indique clairement qu'il s'agit d'un objet matériel (lc poème ou un passage poétique) et non d'un objet formel pour lequel l'affirmation serait valable. 
somme de ses parties. Il s'apercevrait alors que, s'il a eu raison d'accorder à Socrate qu'une série homogène relève d'une science unique, il a eu tort de concéder qu'un objet quelconque ne peut relever que d'une seule science, étant donné qu'il peut parfaitement relever de deux sciences différentes selon qu'on le considère sous des aspects différents. Le sophisme de Socrate consiste à affirmer de chacune des parties d'un tout ce qui est vrai du tout. Au lieu d'accepter que, pour l'étude d'un poème, on fasse de la j’ $\alpha \cdot \psi \omega \delta: x \dot{r}_{1}$ une science mise sur le même pied que la divination, la médecine, etc., Ion devrait évidemment exiger qu'elle soit reconnue hétérogène, et, dans le cas présent, supérieure à toutes les autres, puisque son objet est un ensemble considéré comme tel. Les autres sciences peuvent faire comparaître un à un chacun des matériaux du poème; elle juge du poème en tant qu'il est une unité. Mais Socrate a si soigneusement identifié l'œuvre avec la somme de ses matériaux qu'lon ne s'y retrouve plus. La mort dans l'âme, il laisse dépecer le poème: le conducteur de chars, le médecin, le devin, etc., jugeront de toutes les scènes où leurs techniques se trouvent évoquées. Ion essaie encore de garder les scènes oú n’apparaît aucune spécialité, celles où parlent le mari et la femme, l'esclave et l'homme libre, celui qui obéit et celui qui commande. Sur ce domaine restreint, Socrate le traque encore. Et si celui qui commande est pilote? si la femme tisse la laine? si l'esclave est un bouvier? Autant de portes par où Ion consent à laisser rentrer les techniciens qui l'expulsent. Finalement, il n'y a plus qu'une seule position à laquelle il se cramponne: il se dit capable de juger les passages où un général exhorte

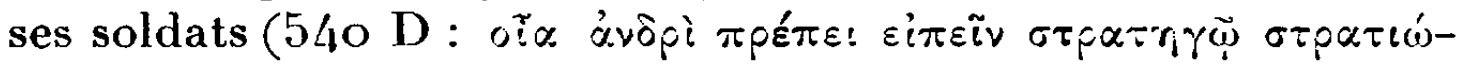
$\tau \alpha ! 5 \pi \alpha_{\rho} \alpha\left(v \circ \bar{\nu}^{\prime} \tau !\right)$. C'est le bon sens même : dans ces exhortations s'expriment uniquement les sentiments communs où Socrate lui-mème a reconnu l'étoffe de toute poésic (53 I $\mathrm{C}$ ). Mais aussitòt Socrate, avec une étonnante mauvaise foi, convertit l'innocente prétention d'Ion en la proposition suivante : ainsi donc, l'art du rhapsode est identique à celui du 
général: lon, qui commence à savoir ce qu'il en coủte de faire des concessions à Socrate, se retranche comme il l'a fait plus haut ( $532 \mathrm{~B}$ et $533 \mathrm{C}$ ) dans une certitude individuelle: ce qui est sùr, c'est que je saurais ce qu'un stratège doit dire. Mais Socrate, qui ne se tient pas satisfait, le fatigue si bien qu'il lui extorque cetle déclaration ridicule : je suis stratège en mème temps que rhapsode et en tant que rhapsode (il consent du reste qu'lon nie la réciproque, à savoir que le stratège, en tant que stratège, comprend les poètes). Et il se garde bien de relever la phrase d'Ion, qui ajoute avoir appris

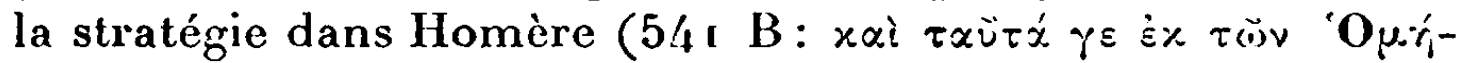
pou ux 0 (i)y, de laquelle on pourrait déduire cette idée raisonnable que le poc̀te, sachant bien des choses, les enseigne à ses lecteurs ${ }^{1}$. Il préfère faire dire à Ion que l'art rhapsodique implique l'art stratégique, ce qui est une évidente absurdité.

Le voici arrivé à son but: la science rhapsodique n'existe pas puisque Ion n'a pu lui découvrir d'objet. On lui a enlevé l'une après l'autre toutes les choses qu'elle croyait posséder en propre. Ion, après s'être laissé expulser de chacune des pages d'Homère, n'a pas songé à affirmer l'existence du poème comme une réalité indivisible relevant d'une forme spéciale de la connaissance; et celte forme spéciale de la connaissance serait précisément celle qu'il possède. Bien cntendu, en affirmant qu'il comprend Homère seul, lon cxcluait d'avance toute identification entre sa forme personnelle d'intelligence poétique et une science quelle qu'elle furt. A cela, il était résigné depuis le début, mais il n'était nulle-

I. L'éducation par les poètes est une des thèses de Protagoras que Socrate rejettc le plus nettement. Ce qu'ont dit nos prédecesseurs ne compte pas : chacun de nous doit chercher la vérité pour son compte :

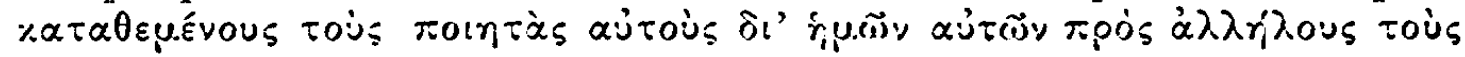

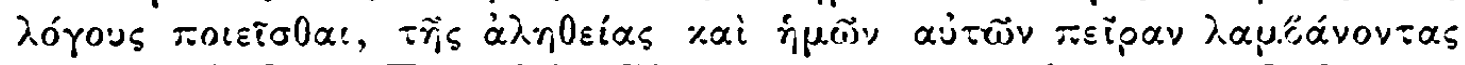
(Protag. 348 A). Toutefois, Platon ne contesterait pas que la lecture des poètes ne puisse aider à saisir d'humbles réalités contingentes. la phrase d'Ion, qui conduirait à un développement nouvcau. celui qni est traité dans Protagoras, n'est pas relcvéc. 
ment résigné à voir nier l'existence même de cette sorte d'intuition poétique où il voit très justement une réalité sui generis.

Le dialogue se termine par une jolie épigramme. Si tu possèdes une scicnce, dit Socrate, tu m'as trompé, car tu as refusć de m'en montrer l'objet. Si vraiment il t'est impossible de m'en montrer l'objet, c'est que tu participes, non à une science, mais à une inspiration divine. En consé-

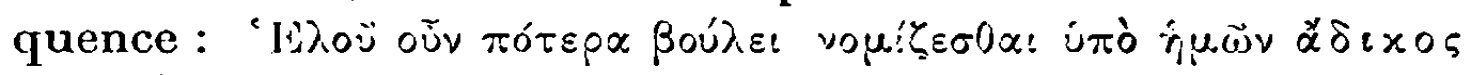

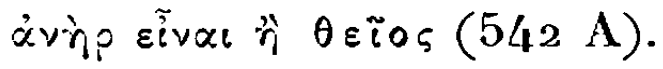

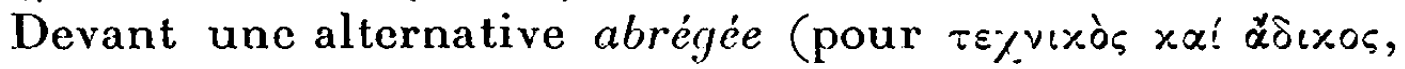

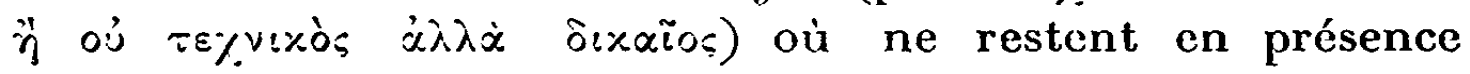
qu'un terme défavorable et un terme flatteur, la vanité d'Ion n'bésite pas. 11 choisit l'explication la plus avantageuse, uniquement parce qu'elle lui paraît plus agréable : $\pi 0 \lambda i \delta$ is $\delta$

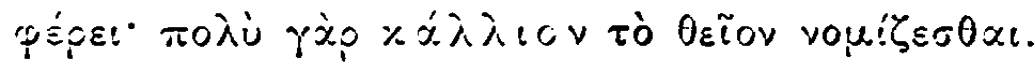

Socrate sourit : inutile d'expliquer à Ion qu'il ne s'agit pas de choisir le plaisant, mais le vrai; Ion ne comprendrait pas. Seulement, dans la conclusion, le dangereux sophiste juxtapose malicieusement le terme flatteur et son équivalent

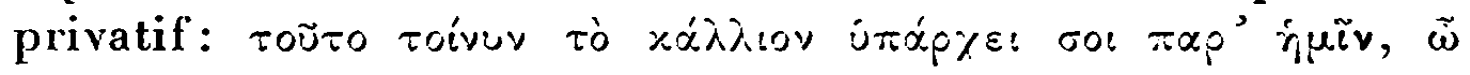

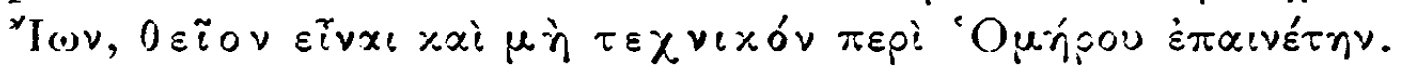

C'est rappeler à Ion, avec une certaine cruauté, les concessions coùteuses qu'il vient de faire.

Marie Delcourt. 